F

\title{
Modelling Bicycle Infrastructure in SUMO
}

\author{
Georgios Grigoropoulos ${ }^{1}$, Leonhard Lücken ${ }^{2}$, Jakob Erdmann ${ }^{2}$ and Heather \\ Kaths ${ }^{1}$ \\ ${ }^{1}$ Technical University of Munich \\ ${ }^{2}$ German Aerospace Center (DLR) \\ georgios.grigoropoulos@tum.de, Leonhard.Luecken@dlr.de, Jakob.Erdmann@dlr.de, \\ heather.kaths@tum.de
}

\begin{abstract}
Bicycle traffic is becoming an increasingly important part of urban traffic. Thus, the simulation and accurate representation of bicycle traffic in microscopic traffic simulation software is gaining importance. As bicycle traffic increases, dedicated bicycle infrastructure is designed to accommodate bicycle traffic. Especially at intersections, the design of intersection approaches follows specific rules and geometric limitations as defined by official design guidelines used in different countries across the world. However, when special environmental factors that affect the intersection layout, such as available space or gradient are not considered, specific standard forms of intersection approaches can be determined based on the number of traffic lanes, the traffic signal control and in the case of this study, the availability as well as the type of dedicated bicycle infrastructure. Categories with available bicycle infrastructure include the cases of bicycle lanes or advisory cycle lanes with advance stop lines for direct left turning bicyclists, the bicycle lanes or advisory bicycle lanes with bicycle boxes and bicycle lanes or bicycle paths with advanced stop lines and a stop area downstream for facilitating an indirect left turn or a two-stage (left) turn of bicyclists. The simulation of such bicycle infrastructure is not natively supported in microscopic traffic simulation software and is mostly only possible through intuitive adjustment of existing network design elements. In this paper, fictional intersections with special bicycle infrastructure are modelled in SUMO. Bicycle traffic data is collected at intersections in Germany with different types of bicycle infrastructure. The collected bicycle traffic data is then used to evaluate the intersection models. Specific recommendations for modelling bicycle infrastructure at intersection approaches in SUMO are provided, and limitations of the proposed methodologies and software limitations are discussed. Results show that the developed solutions can be used to model the bicycle traffic behavior with a reasonable degree of accuracy only for simulation scenarios and traffic situations unaffected by the identified software limitations.
\end{abstract}




\section{Motivation}

Bicycle traffic is becoming an increasingly important part of urban traffic. As bicycle traffic in urban areas increases, traffic engineers and public authorities introduce and design new types of bicycle infrastructure to accommodate the increasing share of bicycle traffic in urban areas. Such infrastructure includes bicycle paths, dedicated bicycle lanes, bicycle boxes. or bicycle highways. Due to the intersection layout, signal control and the expected traffic volume, a wide variety of interactions between bicyclists and motor vehicle traffic can be observed at signalized intersections. These interactions can strongly affect the traffic efficiency at signalized intersections, particularly at intersections with high volumes of bicycle traffic. Thus, the inclusion and consideration of bicycle traffic during the design of traffic infrastructure or traffic management strategies is becoming more important. In order to assess different intersection design scenarios, tools are necessary that not only consider the effects of bicyclists on overall traffic efficiency, but also the effects of different types of bicycle infrastructure and bicycle traffic signal control as well. Examples of such special infrastructure for intersection approaches may include bicycle boxes, advanced stop boxes or advanced stop lines. These can be installed at intersection approaches in order to position bicycles in front of motorized vehicles, increase visibility, improve the bicycle traffic flow and reduce conflicts with right turning motorized vehicles.

Currently available microscopic traffic simulation tools are very limited in regards to native support or provision of specific guidelines on how to model special bicycle infrastructure. Microscopic traffic simulation software such as AIMSUN, SUMO and PTV Vissim can model bicyclists and their respective interactions with other users to a certain extent (Twaddle, Schendzielorz, \& Fakler, 2014). When it comes to special bicycle infrastructure such as bicycle boxes, no specific solutions from the respective software are provided. In such cases users are left with an experimental approach of adjusting existing network design elements and modifying network elements, road user restrictions, traffic signal control elements, bicyclist behavior, or even script solutions through the respective API to more accurately model the respective intersection layout, traffic control and road user behavior. Such solutions come with a toll on model accuracy, quality, and reproducibility, as the respective software does not natively support the functionalities.

The introduction of functions in microscopic traffic simulation software that accurately model bicycle infrastructure and the respective traffic participant behavior is therefore of high importance in order to keep up with the growing share of bicycle traffic. Accurate representation of bicycle infrastructure is necessary for modelling bicycle traffic behavior more realistically as bicyclist behavior is also influenced since the available bicycle infrastructure influences bicyclist behavior. Additionally, it is a precondition for a meaningful simulative comparison of different intersection designs considering dedicated infrastructure elements for bicyclists, because the interactions of motor vehicles and bicyclists need to be depicted accurately to evaluate traffic efficiency and safety for the different cases.

This paper presents a methodology for modelling bicycle infrastructure in SUMO (Lopez et al., 2018). First, the study cases for intersection approaches with bicycle infrastructure are defined, which correspond to the German Design Guidelines (FGSV, 2006b, 2010). Weaknesses of SUMO in modelling specific bicycle infrastructure are identified. Then, new software features and modifications introduced in SUMO in order to facilitate the modelling of bicycle infrastructure are described. Furthermore, the methodology for designing and modelling special bicycle infrastructure in SUMO is described and recommendations for appropriately adjusting the road user behavior are in some cases provided. The developed simulation models are then calibrated based on video data of road users that was collected in Berlin and Munich at two signalized intersections during the summer months of 2017. Finally, restrictions in the proposed methodology are identified and discussed, and recommendations on future SUMO extensions and features are provided. 


\section{Methodology}

\subsection{Definition of Intersection Approaches and Review of SUMO Modelling Capabilities}

Intersection approaches are designed following specific guidelines, which specify the geometry based on the traffic demand to ensure traffic efficiency and traffic safety. Here, specific standard forms of intersection approaches are defined based on the number of traffic lanes, the traffic signal control and the availability as well as the type of dedicated bicycle infrastructure. In the German Design Guidelines (FGSV, 2006b, 2010), five categories of intersection approaches for bicycle traffic are identified:

- Intersection approaches with no dedicated bicycle facility (Category 1)

- Obligatory or advisory cycle lanes with advanced stop lines (Category 2)

- Obligatory or advisory cycle lanes with bicycle boxes (Category 3)

- Bicycle lanes (Category 4) or bicycle paths (Category 5) with advanced stop lines and a stop area downstream for accommodating indirect left turning bicyclists

The existing types of intersection approaches with respect to bicycle infrastructure are depicted in Table 1.

Using the SUMO tool NETEDIT, it is possible to model road and traffic control infrastructure in SUMO. NETEDIT provides several possibilities for customizing the geometric shape of edges, traffic lanes and junctions as well as defining road user type dependent permissions. It is currently possible to model category 1 intersection approaches and approach types 2.1 and 2.2 of category 2 directly in SUMO without further adaptation of the network elements or the software itself. Approach types 2.3 to 2.6 facilitate direct left turning maneuvers for bicyclists except for intersection approach 2.5 , where the bicycle lane should be used only by crossing bicycle traffic. Motorized vehicles turning left or right at the intersection can cross over the respective cycle lanes. A problem arises here in the simulation with right turning motor vehicles crossing the bicycle lane. SUMO provides the possibility to design traffic lanes on edges with different permissions for specific vehicle types. However, it is not possible for motor vehicles to cross over lanes that are only allowed for bicycles. Allowing all vehicle types to cross the bicycle lanes proved to be problematic in, in which motor vehicles were observed to occupy and drive over the bicycle lane rather than just cross it. Thus, specific solutions had to be developed in order to model the behavior of all road users more accurately.

Category 3 includes intersection approaches with bicycle boxes. Bicycles are expected to stop inside the bicycle box area and in the case of left turning bicyclists, ride over to the bicycle box area and align on the left side. Currently, there is no native solution for modelling a bicycle box and the respective road user behavior in SUMO. Additionally, bicyclists should align themselves at the intersection approach depending on the maneuver they intend to perform, which is currently not supported in SUMO. Moreover, the Traffic Light Model in SUMO does not provide the possibility to control only specific road user types entering a junction. Thus, a simple positioning of two signal control positions for the motor vehicle users and the bicycles respectively in order to define the limits of a bicycle box is not possible. The proposed solutions are supported by new introductions in the SUMO Model for enabling realistic road user behavior. 


\section{Bicycle infrastructure categories}

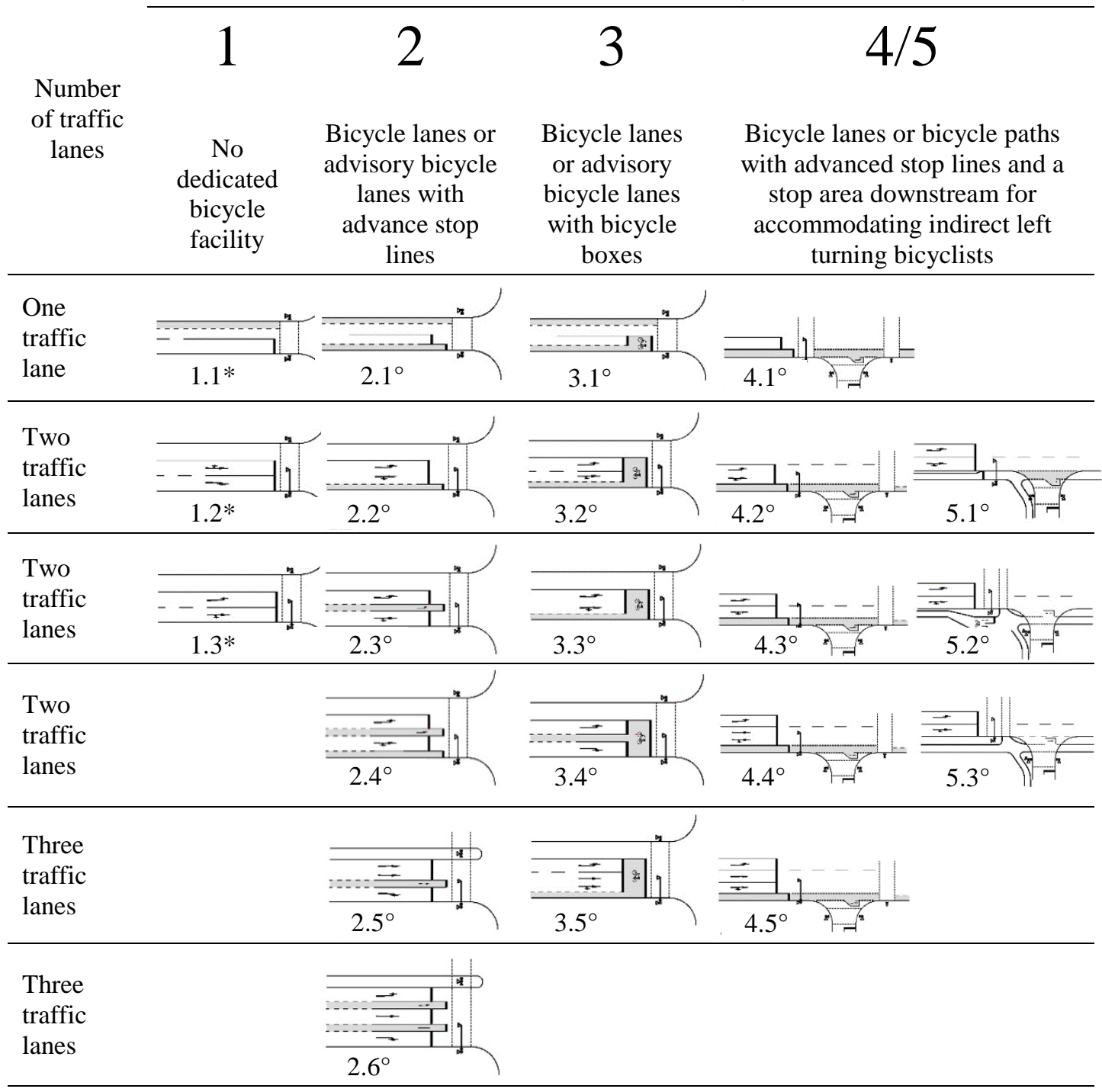

* Overtaking of stopped motorized traffic depends on traffic lane width

${ }^{\circ}$ Dedicated signalization for bicycle traffic possible

Table 1: Bicycle infrastructure categories

Categories 4 and 5 include bicycle lanes or bicycle paths with advanced stop lines and a stop area downstream for facilitating an indirect left turn (FGSV, 2010) also referred to as a two-stage turn (National Association of City Transportation Officials, 2014) for bicyclists. The design of bicycle lanes and bicycle paths is natively supported in SUMO. Additional adaptations of the junction, edge or lane geometry are also possible for the user. However, the simulation of a downstream stop area is not possible in SUMO, either with special network elements or with the existing SUMO Model functions. The process used to model three typical examples for the categories 2, 3 and $4 / 5$ are thoroughly described in this paper. 


\subsection{Enhancements of the SUMO Software}

Several enhancements have been introduced to various components of the SUMO simulation's Junction Model (Erdmann \& Krajzewicz, 2013; Lopez et al., 2018). To allow for separate stopping positions for motorized vehicles and bicyclists, a new parameter stopOffset for network edges (lanes) has been introduced. Theoretically, the implemented data structure makes it possible to define a different stopping position for each SUMO vehicle class by adding according child elements to a lane or edge element as shown in Figure 1.

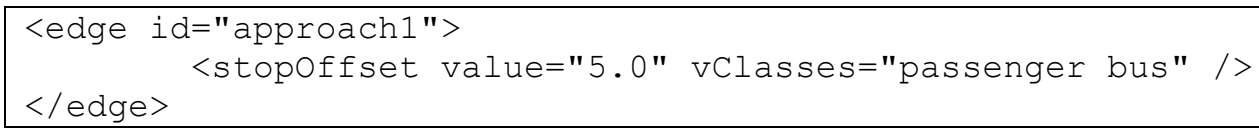

Figure 1: Example for the definition of a stopOffset-element, specifying a stopping position for passenger vehicles and busses located $5 \mathrm{~m}$. in front of the edge's endpoint. Analogously, stopOffset-elements can be defined for single lanes.

A stopOffset-element may be employed to prevent motor vehicles from entering bicycle boxes within a red phase of the corresponding traffic light (Bicycle infrastructure category 3, Table 1) and to represent distinct stopping lines for different vehicle classes or on different lanes on the same edge (Bicycle infrastructure category 32,4 , and 5, Table 1). Vehicle classes considered by a stopOffsetelement and still queueing inside the stopOffset distance at the end of the red time will ignore the red traffic light and flow through the junction. The previously available endOffset attribute for lanes is deprecated since the introduction of stopOffset elements and should not be used anymore.

Another novel modeling component, which is useful to achieve a desirable behavior at intersection approaches, is the lane change model parameter lcTurnAlignmentDistance. This parameter is set as an attribute of the corresponding vType element in the demand configuration. If it is set for a vehicle's vehicle type in this way, it controls the alignment behavior during the approach to an intersection for the given distance prior to the intersection entry. In effect, a vehicle, which is planning to take a left turn, will orient itself laterally towards the left boundary of the turning lane, while right turning vehicles prefer staying at the right boundary and straight going vehicles are not affected. The latter keep their configured or the default alignment behavior. This mechanism is an important ingredient for the modeling of bicyclists behavior at bicycle boxes as it induces a turn specific distribution of the bicycles within the box. If this behavior is not activated, we observed undesirable difficulties for bicyclists entering the box in the first place, or leaving it during a green phase.

\subsection{Modelling Bicycle Infrastructure in SUMO}

Three fictional intersections are designed in CAD with realistic dimensions and are imported in SUMO. Specifically, intersection types 2.5, 3.2 are modelled to describe the modelling procedure of intersections of categories 2 and 3. These intersection approach types are frequent design forms used in urban intersections. Additionally, empirical data for the bicycle traffic have been collected for these specific intersection approach types that can be used for the calibration of the bicycle traffic behavior. Finally, intersection approach type 4.3 is modelled only for the purpose of describing the steps to simulate downstream stop areas used for indirect left turning maneuvers by bicycle traffic. In all examples the Sublane Model of SUMO is utilized which allows vehicles to take out lateral maneuvers within a single lane and thus bicycle traffic behavior more realistically. The use of the Sublane Model requires additional adaptations and the implementation of traffic lane restrictions in some of the proposed solutions. 
Intersection approach type 2.5 is used here as an example of how to model intersection approaches with bicycle lanes or advisory bicycle lanes and advance stop lines (category 2) in SUMO using NETEDIT (Figure 2). This intersection approach consists of two traffic lanes for crossing motor vehicle traffic, one lane for crossing bicycle traffic and one traffic lane for right turning motor vehicle traffic. The intersection approach model in SUMO consists of four traffic lanes, which is equal to the number of traffic lanes found in the standard intersection approach form. The intersection approach in SUMO consists of two separate edges. This separation is implemented so that motor vehicles turning left at the intersection approach can cross over the bicycle traffic lane shortly before the intersection using the dedicated left turning lane. Edge 2 is subsequently modelled with traffic lane 3 as a dedicated bicycle traffic lane, as motor vehicles were often observed driving or stopping on traffic lane 3 , which was intended for use only by bicycle traffic. This unrealistic behavior was occurring due to the use of the Sublane Model. The length of Edge 2 can be further adjusted by the modeler with respect to real traffic observations of driving maneuvers of left turning vehicles. In cases where a dedicated left turning lane for bicycle traffic is introduced a short distance before the intersection area, it is suggested that an intermediate edge is positioned between Edge 1 and 2 with the same traffic lane restrictions as Edge 2 to facilitate the lane changing maneuvers of bicycle traffic intending to turn left at the intersection. Finally, the advanced stop line of the bicycle lane is best modelled through customizing the geometry of the junction area.

\section{Intersection Approach Type 2.5}

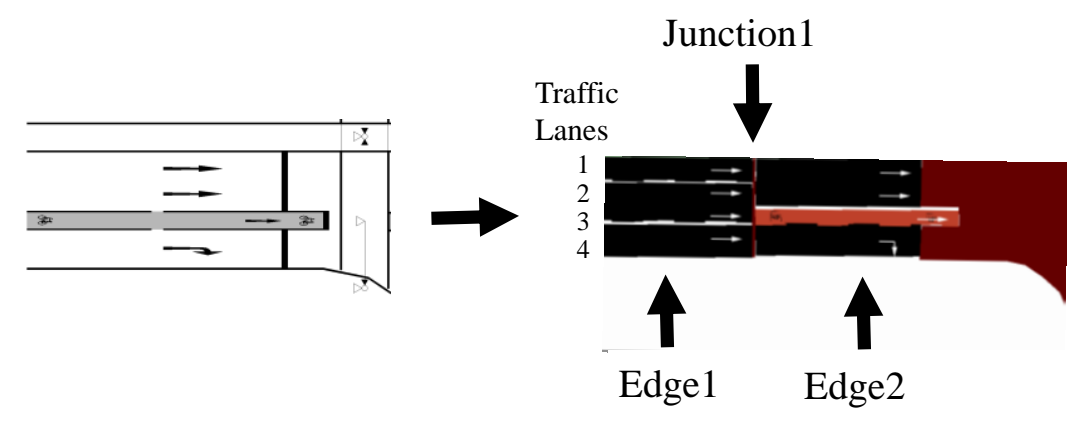

Figure 2: Modeling intersection approach Category 2 in SUMO (example: type 2.5)

Intersection approach type 3.2 is used as a generalized example of how to model intersection approaches with bicycle lanes or advisory bicycle lanes and bicycle boxes (category 3 ) in SUMO using NETEDIT (Figure 3). This intersection type is modelled with two subsequent Edges 1 and 2. Edge 1 is designed with the desired number of traffic lanes for each type of road user. In this case, three lanes are designed, two for motor vehicle traffic and one for bicycle traffic. Edge 2 is designed with one common traffic lane for all road users, a width equal to the sum of widths of the traffic lanes of Edge 1 and length equal to the length of the simulated bike box. It is important to match of the width of the lane representing the bicycle box and the cumulative width of the predecessor lanes exactly, since this triggers a heuristic that adapts the appropriate positioning of connections. Junction 1 is simulated as an unsignalised intersection. The realistic traffic behavior for a bicycle box is then modelled with the simulation parameters stopOffset and lcTurningAlignementDistance. In this way, a bicycle box is generated through prohibiting motor vehicles from stopping at a distance that is less than the length of the bicycle box. The empty space created by the stopOffset is then used by stopping bicycles that flow into the bicycle box section. The lcTurningAlignementDistance parameter then forces bicyclists to align themselves laterally at a traffic lane according to their travel direction at the intersection downstream. Finally, the modeler should carefully adjust the starting position of the connections of the intersection approach for all road users. 
Intersection Approach Type 3.2

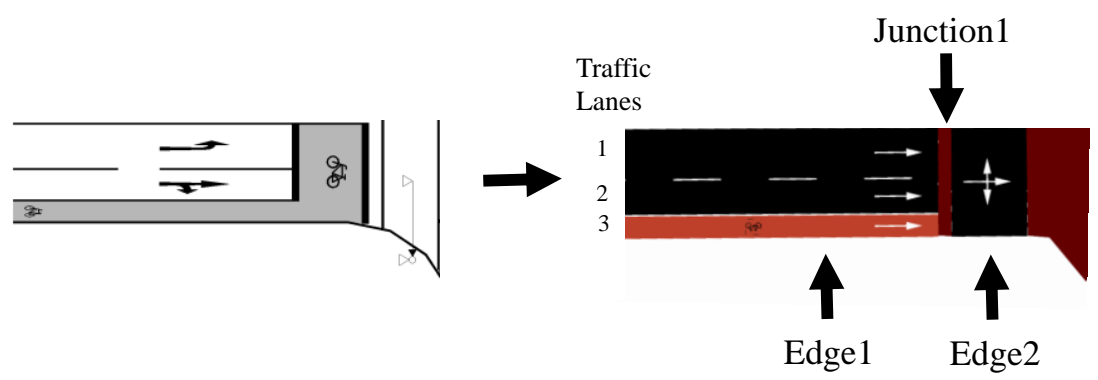

Figure 3: Modeling intersection approach Category 3 in SUMO (example: type 3.2)

Intersection approach type 4.5 is used as an example to model intersection approaches with bicycle lanes or bicycle paths with advanced stop lines and a stop area downstream for accommodating indirect left turning bicyclists (category 5) in SUMO using NETEDIT (Figure 4).

Intersection Approach Type 4.4

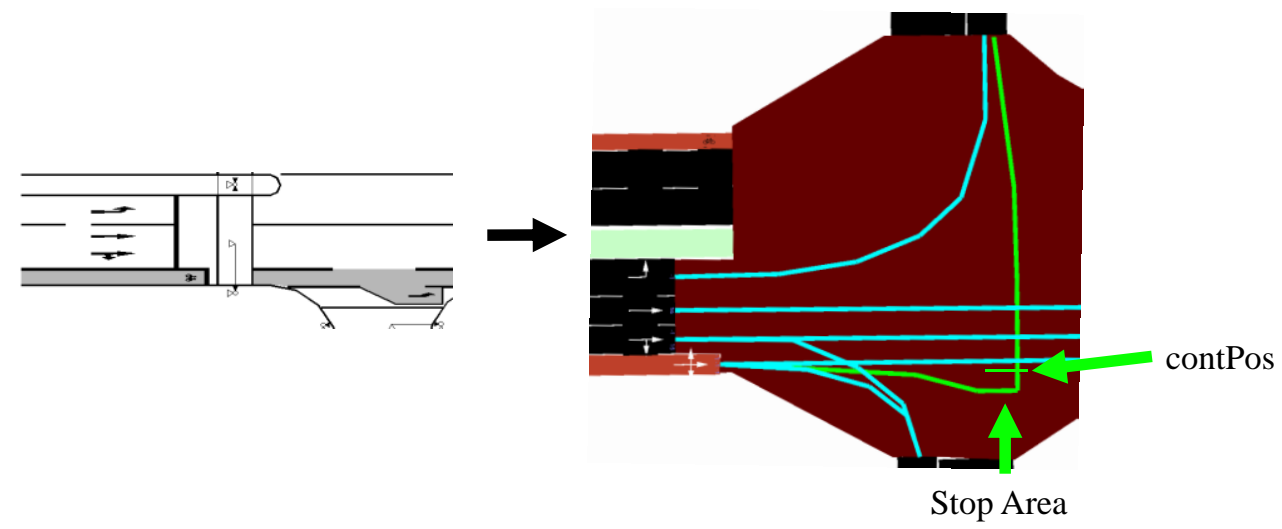

Figure 4: Modeling intersection approach Category 4/5 in SUMO (example: type 4.5)

Modelling this type of traffic control for bicycle traffic requires the modeler to edit the network xml file of the SUMO network. Figure 5 presents an example for editing the network file of a SUMO network in order to simulate indirect left turning behavior of bicycle traffic. Specifically, the modeler has to locate the connection in the SUMO network file that will be used for indirect connecting the internal junction (Erdmann \& Krajzewicz, 2013) corresponding to the intermediate stopping position of the left turning bicycles with the subsequent lane behind the junction. Two additional attributes must be added to the xml definition of the connection so that road users driving on the connection are controlled by a different phase of the traffic control at the intermediate stopping position. $t l$ is added to the xml definition and its value is equal to the junction id. The linkIndex is also added to the xml definition and defines the signal group at the intermediate stopping position contPos. By adding this definition, traffic driving on the connection is regulated by the traffic signal control of the junction at the user defined intermediate stopping position. Finally, the modeler has to customize the geometry of the connection and edit the intermediate stopping position in order to realistically model the trajectory of indirect left turning bicycle traffic. It is important to mention here that in case of changes of the 
model in NETEDIT, the modeler's changes for indirect turning are overwritten. Thus, it is advised that the modeler introduce these changes in the xml file as a last step during the network design process.

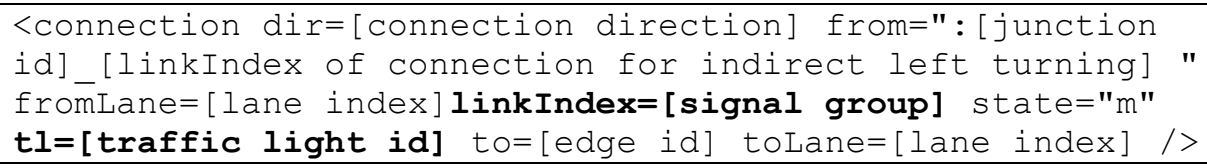

Figure 5: Example for the editing a connection in a SUMO network file in order to simulate indirect left turning behavior of bicycle traffic. Bold font highlights the new attributes necessary for simulating indirect left turning behavior.

\subsection{Modelling Solution Restrictions}

The solutions presented in Section 2.3 come with certain restrictions with respect to their implementation and the realistic simulation of the behavior of road users.

Restrictions apply concerning the modelling of lane changing behavior and the behavior of bicyclists inside the junction area in category 3 . Left turning bicyclists will not make use of the traffic lanes for motor vehicle traffic to align themselves on the left side of the bicycle box if the traffic situation at the intersection approach permits it and will always access the bicycle box through the bicycle lane. This issue can be resolved either by defining two user groups for left turning bicyclists one making use of the bicycle lane to approach the bicycle box and one that is making use of the motor vehicle traffic lanes to approach the bicycle box. Changing this behavior depending on the traffic state at the bicycle box is then only possible using TraCI. Another observed issue in category 3 are the interactions between right turning and crossing bicyclists inside the bicycle box. It is often observed at real intersection with bicycle boxes (approach types 3.2 and 3.3) that bicyclists crossing or turning right only make use of the right side of bicycle box and do not distribute themselves across the entire width. This behavior creates a queue of bicyclists that effectively blocks access to the bicycle box area. In SUMO, right turning bicyclists sometimes align themselves on the left side of a bicyclist riding straight across the intersection while remaining on the right side of the bicycle box. This bicyclist tends to block crossing bicyclists once the green phase begins.

Finally, in category 3 , driving behavior in the connectors of the junction is not always realistic due to the fundamental design of the Junction Model mainly for left turning traffic users. Under real traffic conditions, left turning bicyclists will make use of available space of the inner intersection area adapting to the traffic situation. They will distribute themselves making use of free available space among other waiting vehicles to reduce the size of the acceptable time gap between prioritized vehicles. SUMO's Junction Model cannot support this degree of flexibility among road users. Road users are obliged to travel on the connector only and effectively block other road users. Thus, this concurrent use of the road space cannot be simulated.

Restrictions in the modelling of indirect left turning behavior apply mainly due to the inherent flexibility of bicyclists. Queuing bicyclists will distribute themselves inside the stopping box area or around it without blocking bicyclists crossing the intersection of the same approach. The simulation of this complex bicyclist behavior in SUMO is not possible and would require a significant expansion of the Junction Model.

\section{Simulation}

Two fictional intersections are modelled in SUMO. Two intersection approaches of the same category are modelled in each intersection and the modelled intersection approaches belong to type 2.5 
and to type 3.1 respectively. Traffic signal programs are calculated for both intersection types according to the HBS (FGSV, 2015) using motor vehicle traffic volumes resulting in a minimum Level of Service (LOS) B. First the modelled intersections are calibrated only for motor vehicle traffic according to the recommendations of (FGSV, 2006a) using the average waiting time as the measure of performance. Subsequently, bicycle traffic is introduced to the simulated intersections. Four simulation studies with increasing bicycle traffic flows are conducted in order to vary the number of arriving and stopping bicyclists in each cycle time. Each cycle time has a duration of 90 seconds and a total of 150 cycle times are evaluated for each intersection. Due to the limitations described in Section 2.3, right turning bicycle traffic is not considered on intersection approach 3.1.

Simulated bicycle traffic is calibrated and validated using data collected at three intersection approaches of category 2, one in Berlin (type 2.5), two in Munich (type 2.5) and one intersection of category 3 in Berlin (type 3.1).

For all intersections, two-hour video segments with a particularly high volume of bicycle traffic were selected. The trajectories of the road users were extracted using two different methodologies, as the video data collection was split between two research partners. The Munich, trajectories were extracted from the video data using Traffic Intelligence (Saunier \& Sayed, 2006). The Berlin data were analyzed by the German Aerospace Center (DLR) utilizing an internally developed software.

Bicycle traffic behavior is calibrated by adjusting the car-following and lane-changing model attributes in SUMO and using the bicycle queue density as the measure of performance for the calibration and the average queue dispersion time of bicyclists as the measure of performance for the validation in the case of intersection approach 2.5 . In the case of the intersection approach type 3.1 the quality of the simulation is only assessed in terms of the observed average queue dispersion time as the density of bicycle traffic is a function of the fixed dimensions of the bicycle box. Thus, the queue density value is fixed for the same number of bicyclists inside for the same bicycle box. The bicycle queue density for intersection approach 2.5 is estimated using the following equation:

$$
\mathrm{k}_{\mathrm{b}, \text { queue }}=\frac{\mathrm{n}_{\text {stop }}}{\mathrm{l}_{\text {queue }} \mathrm{b}}
$$

Where:

$\mathrm{k}_{\mathrm{b}, \text { queue }}=$ Bicycle queue density at a bicycle path, bicycle lane or advisory bicycle lane [bicycle/m2]

$\mathrm{n}_{\text {stop }}=$ Number of bicyclists queued at the start of the green time [-]

$1_{\text {queue }}=$ Bicycle queue length at the start of the green time $[\mathrm{m}]$

$b=$ width of the bicycle infrastructure [m]

The average queue dispersion time of bicyclists is calculated as the time of the last bicyclist in queue divided by the number of queued bicyclists: The following equation is used to derive the average time headway in every cycle time.

$$
t_{d t, b i c y c l e}=\frac{t_{n}}{n}
$$

Where:

$t_{\mathrm{dt}, \text { bicycle }}=$ average queue dispersion time $[\mathrm{s}]$

$\mathrm{t}_{\mathrm{n}}=$ Time from the start of the green time until the last $\left(\mathrm{n}^{\mathrm{th}}\right)$ bicyclist crosses the stop line [s]

$\mathrm{n}=$ Number of bicyclists waiting at the start of the green time [-]

Finally, Welch's t-test is used to compare the simulated and the observed data and assess the accuracy of the simulation. 


\section{Results}

The results of the simulation for the two intersections are presented in Figure 6 for intersection approach type 2.5 and Figure 7 for intersection approach type 3.1. The simulation results are analyzed using Box-Whisker diagrams.
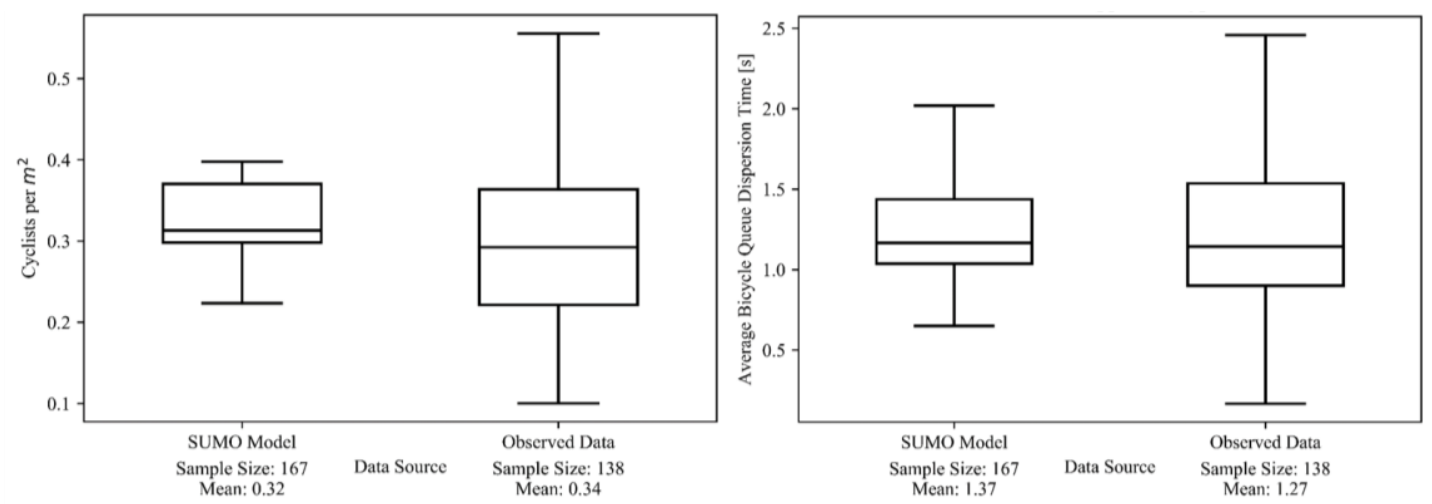

Figure 6: Comparison between the SUMO Model and the observed data using the bicycle queue density average queue dispersion time for bicyclists at the intersection approach type 2.5

The SUMO Model provides a smaller deviation of the density and the average queue dispersion time in comparison to the observed data for intersection approach 2.5. However, the estimated average values of both performance measures are very close to the ones of the observations.

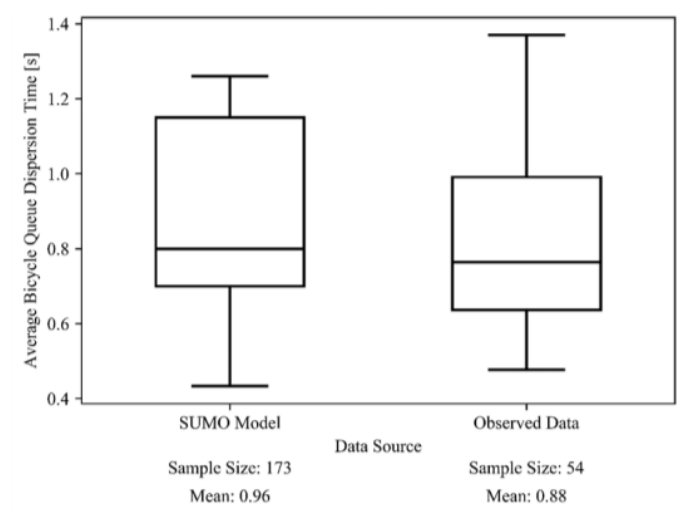

Figure 7: Comparison between the SUMO Model and the observed data using the average queue dispersion time for bicyclists at the intersection approach type 3.1

In contrast to the results of intersection approach type 2.5, the SUMO Model provides a wider range of possible average queue dispersion time values in the case of intersection type 3.1 than the ones observed in reality. The slightly higher average time values of the SUMO Model may in this case be the result of the Junction Model's inability to replicate the flexibility of bicycle traffic in intersection approaches and the more efficient use of available road space. Thus, the average dispersion time is higher.

Welch's t-test is used to compare the means of simulated and the observed assess the accuracy of the simulation. The results are presented in Table 2. Welch's t-test is performed for the null hypothesis 
that the independent samples (simulation data and observation data) have identical average (expected) values assuming unequal variances.

\begin{tabular}{cccccccc}
\hline $\begin{array}{c}\text { Intersection } \\
\text { Approach } \\
\text { Type }\end{array}$ & $\begin{array}{c}\text { Measure of } \\
\text { Performance }\end{array}$ & $\begin{array}{c}\text { Mean } \\
\text { Observations }\end{array}$ & $\begin{array}{c}\text { Mean } \\
\text { Simulation }\end{array}$ & $\begin{array}{c}\text { Percent } \\
\text { Error } \\
\text { (PE) }\end{array}$ & $\begin{array}{c}\text { p- } \\
\text { value }\end{array}$ & $\alpha$ & $\mathrm{H}_{0}$ \\
\hline 2.5 & $\begin{array}{c}\text { Bicycle Queue } \\
\text { Density }\end{array}$ & 0.32 & 0.34 & $7.0 \%$ & 0.16 & 0.05 & accepted \\
2.5 & $\begin{array}{c}\text { Av. Bicycle Queue } \\
\text { Dispersion Time } \\
\text { Av. Bicycle Queue } \\
\text { Dispersion Time }\end{array}$ & 1.37 & 1.27 & $7.4 \%$ & 0.17 & 0.05 & accepted \\
\hline
\end{tabular}

Table 2: Statistical analysis results

Since p-value $>\alpha, H_{0}$ is accepted for simulation studies. The average of the simulation's population is considered to be equal to the average of the observation's population. Thus, the simulation was able to reproduce the traffic situations observed at the real intersection approaches accurately.

\section{Conclusions}

In this paper, a methodology for simulating bicycle infrastructure in SUMO is presented and evaluated using traffic efficiency indicators as measures of performance. Results show that the proposed modelling solutions and new functions introduced to SUMO can be used to simulate bicycle traffic using dedicated bicycle infrastructure. The proposed methodology is, however, applicable only to specific traffic scenarios mainly due to limitations of the SUMO Junction Model and since the proposed solutions still do not rely on dedicated SUMO network design elements specifically designed to accommodate simulated bicycle traffic (Section 2.3). Nevertheless, the developed methodology relies on natively supported solutions by the simulation software, which in turn reduces the workload and effort for the modeler. Future extensions of the SUMO Model are planned to modify the lateral gap requirements for bicyclists depending on their speed in order to simulate the bicycle queue density more realistically. Also, the potential of utilizing existing provisions in the SUMO Model for preferred lane usage, which are currently not in use, will be investigated. These might potentially enable motor vehicles to change across bicycle lanes and bicycles to use vehicular lanes to enter the modelled bicycle facilities such as advanced stop lines of bicycle boxes. Finally, further additional software development can improve the strength and quality of SUMO in simulating non-motorized user behavior and provide a valuable tool for further research in understanding the effects of the interactions non-motorized and motorized users on traffic efficiency and safety with respect to special urban road infrastructure.

\section{References}

Erdmann, J., \& Krajzewicz, D. (2013). SUMO 's Road Intersection Model. Lecture Notes in Computer Science (Vol. 8594). Berlin, Heidelberg: Springer.

FGSV. (2006a). Hinweise zur mikroskopischen Verkehrsflusssimulation -Grundlagen und Anwendung. Köln: Forschungsgesellschaft für Straßen- und Verkehrswesen (Hrsg.).

FGSV. (2006b). Richtlinien für die Anlage von Stadtstraßen (RASt). Köln: Forschungsgesellschaft für Straßen- und Verkehrswesen (Hrsg.). 
FGSV. (2010). Empfehlungen für Radverkehrsanlagen (ERA) (2010th ed.). Köln: Forschungsgesellschaft für Straßen- und Verkehrswesen (Hrsg.).

FGSV. (2015). Handbuch für die Bemessung von Straßenverkehrsanlagen (HBS). Köln: Forschungsgesellschaft für Straßen- und Verkehrswesen (Hrsg.).

Lopez, P. A., Behrisch, M., Bieker-walz, L., Erdmann, J., Fl, Y., Hilbrich, R., ... Wießner, E. (2018). Microscopic Traffic Simulation using SUMO, 2575-2582.

National Association of City Transportation Officials. (2014). Urban Bikeway Design Guide. Island Press.

Saunier, N., \& Sayed, T. (2006). A feature-based tracking algorithm for vehicles in intersections. In Third Canadian Conference on Computer and Robot Vision. IEEE.

Twaddle, H., Schendzielorz, T., \& Fakler, O. (2014). Bicycles in Urban Areas: Review of Existing Methods for Modeling Behavior. Transportation Research Record: Journal of the Transportation Research Board, (2434), pp 140-146. https://doi.org/10.3141/2434-17 